\title{
Atoms as nonlinear mixers for detection of quantum correlations at ultrahigh frequencies
}

\author{
N. Ph. Georgiades, E. S. Polzik, * and H. J. Kimble \\ Norman Bridge Laboratory of Physics, 12-33, California Institute of Technology, Pasadena, California 91125
}

(Received 16 September 1996)

\begin{abstract}
Measurements of quantum correlations are reported for a frequency difference of $25 \mathrm{THz}$ between the signal and idler output fields generated by a subthreshold nondegenerate optical parametric oscillator. By simultaneously exciting a two-photon transition in atomic Cs by a combination of signal, idler, and "references oscillator" fields, we record modulation of the excited-state population due to quantum interference between two alternative excitation pathways. The observed phase-sensitive modulation is proportional to the correlation function $\left\langle\hat{E}_{s} \hat{E}_{i}\right\rangle$ for the quantized signal and idler fields. [S1050-2947(97)50303-9]
\end{abstract}

PACS number(s): 42.50.Dv, 42.50.Ct, 42.65.Hw

Phase-sensitive detection of nonclassical correlations of the electromagnetic field has been realized in numerous experiments [1], with measurement of squeezing via homodyne detection [2] now being a well established technique. In principle such quantum correlations should exist for extremely large frequency separations between the correlated signal and idler beams generated by parametric down-conversion. Indeed, for a triplet of frequencies $\left(\omega_{p}, \omega_{s}, \omega_{i}\right)$ for the pump, signal, and idler fields (with $\omega_{p}=\omega_{s}+\omega_{i}$ ), nonclassical correlations manifest at frequency separation $\Delta \omega \equiv\left|\omega_{s}-\omega_{i}\right|$ that itself could in principle be comparable to the optical frequency, $\Delta \omega \leqslant \omega_{p}$. However, for almost all experiments to date, the offsets for the detection of quantum fluctuations of quadrature amplitudes (i.e., squeezing) have been such that $\Delta \omega / \omega_{p} \ll 1$, with a notable exception being ultrafast measurements with femtosecond pulses [3]. By contrast, for nonnegligible values of the normalized offset $\Delta \omega / \omega_{p}$, the fundamental form of the quadrature amplitudes is predicted to change $[4,5]$. However, it is a challenging experimental task to generate and to detect such correlations given that existing photodetector technology has a frequency cutoff of about 10 $\mathrm{GHz}$ that limits detection of $\mathrm{cw}$ quantum fluctuations to $\Delta \omega / \omega_{p} \leqslant 10^{-5}$ for the case of optical frequencies.

In this Rapid Communication we present a detection strategy and evidence of nonclassical correlations associated with quadrature squeezing for signal and idler fields separated by $\Delta \omega / 2 \pi=25 \mathrm{THz}$ with normalized offset $\Delta \omega / \omega_{p} \approx 0.07$. To achieve this ultrahigh frequency measurement, we employ a technique that exploits individual atoms as nonlinear mixing elements for signal, idler, and reference fields [6]. By illuminating three-level atoms simultaneously with three fields $\left(\omega_{s}, \omega_{i}, \omega_{o}\right)$ such that $\omega_{s} \simeq \omega_{12}, \omega_{i} \simeq \omega_{23}$ and $\omega_{o} \equiv \omega_{p} / 2$ $\simeq \omega_{13} / 2$ (Fig. 1), excited-state population $\rho_{33}$ is produced by two alternative pathways, namely, via stepwise dipole transitions $\left(1 \rightarrow 2\right.$ and $2 \rightarrow 3$ ) with fields $\left(\omega_{s}, \omega_{i}\right)$ and via twophoton quadrupole absorption $(1 \rightarrow 3)$ with the reference field $\omega_{o}$. Quantum interference (QI) between the amplitudes of these two excitation paths leads to a phase-sensitive dependence of $\rho_{33}$ that can be exploited to investigate the na-

\footnotetext{
*Permanent address: Institute of Physics and Astronomy, Aarhus University, Aarhus C, DK-8000, Denmark.
}

ture of the quantum fluctuations for the signal and idler fields at $\omega_{o} \pm \Omega_{0}$, where $\Omega_{0} \equiv \Delta \omega / 2$. We note that an important feature of our scheme is that the solid angle of illumination of the atoms by the signal and idler fields is only a few percent, which is contrary to "conventional wisdom" that dictates that large solid angle coverage is required to observe the influence of squeezing on atoms.

To make clear how correlations of the electromagnetic field are mapped to interference in atomic excitation, we describe results of a theoretical analysis based upon the quantum master equation relevant to Fig. 1 and compare our scheme to the more familiar homodyne detection. We also present results from a "proof-of-principle", experiment in which phase-sensitive fluctuations for the quadrature amplitudes are detected for $\Omega_{o} / 2 \pi=12.5 \mathrm{THz}$. At the outset, we note that our technique lacks a readily defined benchmark reference level corresponding to the usual "shot-noise", level for homodyne detection, so that observation of quantum squeezing in its strict form does not apply directly to our measurements, although some possibilities for improving this circumstance are suggested.

The qualitative manner in which QI in an atomic system as in Fig. 1 is utilized to investigate the quantum statistical properties of an illuminating field can be understood by recalling the classic treatment of Mollow [7] for two-photon excitation in which the field correlation function $D(\tau)$ plays a central role. Here,

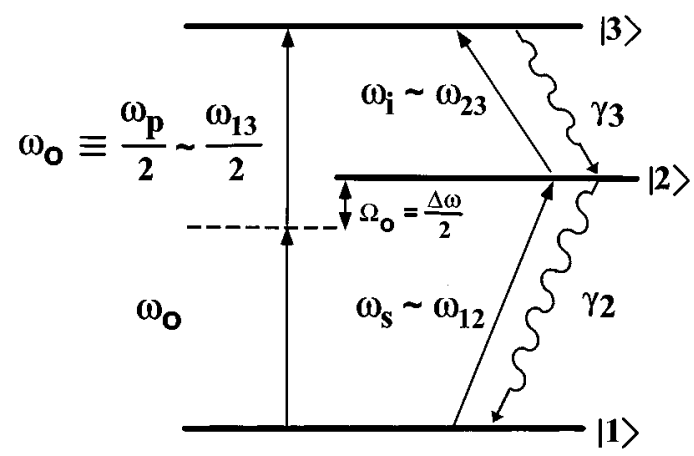

FIG. 1. Energy-level diagram for the atomic system under consideration. 


$$
D(\tau) \equiv\left\langle\hat{E}^{\dagger}(t+\tau) \hat{E}^{\dagger}(t+\tau) \hat{E}(t) \hat{E}(t)\right\rangle,
$$

where now $\hat{E}$ is the total excitation field $\left(\hat{E}=\hat{E}_{\mathrm{RO}}+\hat{E}_{q}\right)$ incident on the atom written in a rotating frame of frequency $\omega_{o}$ (i.e., $\hat{\mathcal{E}}(t)=\hat{E}(t) e^{-i \omega_{0} t}$ ), with the reference oscillator field $\hat{E}_{\mathrm{RO}}$ assumed to be in a coherent state $\left(\left\langle\hat{E}_{\mathrm{RO}}\right\rangle=E_{\mathrm{RO}} e^{-i \beta}\right)$ and the quantum field $\hat{E}_{q}$ assumed to be generated by parametric down conversion. The latter field can be described by $[8,9] \hat{E}_{q}(t)=\int \hat{E}_{q}(\Omega) e^{-i \Omega t} d \Omega$, with $\hat{E}_{q}(\Omega)=\mu(\Omega) \hat{E}_{V}(\Omega)+\nu(\Omega) \hat{E}_{V}^{\dagger}(-\Omega)$, where $\hat{E}_{V}$ is the input field to the parametric device assumed to be in a vacuum state so that $\left\langle\hat{E}_{q}\right\rangle=0$. In the ideal case, the gain coefficients $\{\mu(\Omega), \nu(\Omega)\}$ satisfy the relation $|\mu|^{2}-|\nu|^{2}=1$, with $\mu$ taken to be real while $\mu \equiv|\mu| e^{i \phi}$. The first order correlation functions of $\hat{E}_{q}$ are defined in the usual way be means of the parameters $M$ and $N$ given by

$$
\begin{gathered}
\left\langle\hat{E}_{q}(\Omega) \hat{E}_{q}\left(\Omega^{\prime}\right)\right\rangle \equiv M(\Omega) \delta\left(\Omega+\Omega^{\prime}\right), \\
\left\langle\hat{E}_{q}^{\dagger}(\Omega) \hat{E}_{q}\left(\Omega^{\prime}\right)\right\rangle \equiv N(\Omega) \delta\left(\Omega-\Omega^{\prime}\right),
\end{gathered}
$$

where $M(\Omega)=\mu(\Omega) \nu(\Omega)$ and $N(\Omega)=|\nu(\Omega)|^{2}[10]$. Substituting for $\left(\hat{E}_{\mathrm{RO}}, \hat{E}_{q}\right)$ in Eq. (1) and retaining only terms of order $\left|E_{\mathrm{RO}}\right|^{2}$ and higher, we arrive at the following expression for the Fourier transform $\Phi(\Omega)$ of $D(\tau)$ :

$$
\Phi(\Omega)=E_{\mathrm{RO}}^{4}\left[1+\frac{2 M_{\mathrm{eff}}}{E_{\mathrm{RO}}^{2}} \cos (2 \beta+\phi)\right] \delta(\Omega)+2 E_{\mathrm{RO}}^{2} N(\Omega),
$$

where $\quad \Phi(\Omega) \equiv 1 / 2 \pi \int e^{i \Omega \tau} D(\tau) d \tau \quad$ and $\quad M_{\text {eff }} \equiv$ $\int\left|M\left(\Omega^{\prime}\right)\right| d \Omega^{\prime}$.

By noting that the excited-state population $\rho_{33} \propto \Phi(\Omega)$ [7], we conclude that by monitoring $\rho_{33}$ as a function of the reference oscillator phase $\beta$, a phase-sensitive contribution to $\rho_{33}$ can be identified which is directly proportional to the field correlation function $M_{\text {eff }}$. Unfortunately, although this technique provides a means of measuring $M$, we do not learn anything about $N$. To see why this is the case, note that the first term of Eq. (3) describes the absorption of two photons from $\hat{E}_{\mathrm{RO}}$, while the second term arises from QI between amplitudes for two-photon excitation via the reference oscillator and via the signal and idler fields, where both the first and second terms satisfy the overall two-photon resonance condition and hence contribute to $\rho_{33}$ near-zero frequency (dc terms). By contrast, the last term corresponds to absorption of one photon from the RO and one from either the signal or idler beams, which is far off resonance (Fig. 1) and hence makes a negligible contribution. Stated somewhat differently, for nondegenerate parametric amplification centered around signal and idler frequencies $\omega_{s, i}=\omega_{p} / 2 \pm \Omega_{o}$, the last term in Eq. (3) will be peaked around $\Omega_{o}$, and thus will contribute only negligible high frequency fluctuations to $\rho_{33}$ (in our specific case, $\Omega_{o} / 2 \pi \simeq 12.5 \mathrm{THz}$ ) so that the dominant contribution to $\rho_{33}$ will arise from

$$
\Phi(\Omega) \approx E_{\mathrm{RO}}^{4}\left[1+\frac{2 M_{\mathrm{eff}}}{E_{\mathrm{RO}}^{2}} \cos (2 \beta+\phi)\right] \delta(\Omega),
$$

and hence $M$ but not $N$ can be recovered.

The preceding analysis should be compared to that more commonly encountered for homodyne detection of squeezed light via photoelectric detection, for which the relevant correlation function $C(\tau)$ is defined by [9]

$$
C(\tau) \equiv\left\langle\hat{E}^{\dagger}(t) \hat{E}^{\dagger}(t+\tau) \hat{E}(t+\tau) \hat{E}(t)\right\rangle .
$$

Again taking the total field $\hat{E}=\hat{E}_{\mathrm{LO}}+\hat{E}_{q}$ to be composed of a local oscillator field $\hat{E}_{\mathrm{LO}}$ in a coherent state and the quantum field $\hat{E}_{q}$ as before, we follow the same steps as above to find that the Fourier transform $\Psi(\Omega)$ of $C(\tau)$ is given by

$$
\begin{aligned}
\Psi(\Omega)= & E_{\mathrm{LO}}^{4}\left[1+\frac{2 N_{\mathrm{eff}}}{E_{\mathrm{LO}}^{2}}\right] \delta(\Omega) \\
& +2 E_{\mathrm{LO}}^{2}[N(\Omega)+|M(\Omega)| \cos (2 \beta+\phi)],
\end{aligned}
$$

where $\Psi(\Omega) \equiv 1 / 2 \pi \int e^{i \Omega \tau} C(\tau)$ and $N_{\text {eff }} \equiv \int N\left(\Omega^{\prime}\right) d \Omega^{\prime}$. In contrast to Eq. (3), the term near-zero frequency now carries information about the mean flux of the fields $\left(E_{\mathrm{LO}}, E_{q}\right)$, while a more interesting combination of correlation functions appears in the second term. For fields generated by nondegenerate parametric amplification around $\omega_{s, i}=\omega_{o} \pm \Omega_{o}$, this second term is peaked around $\Omega_{o}$ and describes the (normally ordered) spectrum of squeezing $S(\Omega, \theta)$ for the signal and idler fields, where as usual $S$ is defined by $[8,9]$ $S(\Omega, \theta)=2[N(\Omega)+|M(\Omega)| \cos \theta]$. Thus the well known "good news" for homodyne detection is that $S\left(\Omega_{o}, \theta\right)$ is directly obtained by observing the photocurrent fluctuations at frequency $\Omega_{o}$, while for our proposed scheme, only $M_{\text {eff }} \approx\left|M\left(\Omega_{o}\right)\right| \Delta \Omega_{e}$ enters Eq. (4) with $\Delta \Omega_{e}$ an effective bandwidth for the two-photon transition. On the other hand, the "bad news" for homodyne detection is that information about $S\left(\Omega_{o}, \theta\right)$ is written into the photocurrent at frequency $\Omega_{o}$, which can be well beyond current detector capabilities (e.g., for our work $\Omega_{o} / 2 \pi \simeq 12.5 \mathrm{THz}$ ), while in our scheme, quantum fluctuations described by $M(\Omega)$ are demodulated to near-zero frequency where detection is straightforward. However, to complete the measurement of squeezing, a means must be devised for the direct comparison of $N(\Omega)$ and $M(\Omega)$ as in $S(\Omega, \theta)$. Since $N(\Omega)$ is simply the photon flux of the squeezed field, a "power" measurement should suffice to fix $N$ and hence $S(\Omega, \theta)$, with the non-negligible difficulty being to assure that the measurements of $M$ and $N$ have accurately determined efficiencies, including the details of the optical path in the trap and the diverse atomic parameters for a multistate system. In this regard, the atoms themselves may be employed as "power meters" for $N$ with the atoms illuminated only by the signal beam, resonant with the $1 \rightarrow 2$ transition. The excitation probability $(1 \rightarrow 2)$ thus determined might provide a direct measurement of $N$ with many of the efficiencies common to the measurement of $M$.

To extend this grossly oversimplified discussion to include the relevant details of the atom-field interaction, we have developed a full master equation analysis [11] and briefly present here some of our results. Our starting point is the master equation of Ficek and Drummond [Eq. (20) of Ref. [12(a)]], which is valid for squeezed light of bandwidth much larger than any atomic linewidth or detuning. To this master equation we add a term for the assumed quadrupole 
coupling of the $1 \rightarrow 3$ transition to the coherent-state field $\hat{E}_{\mathrm{RO}}$ of frequency $\omega_{o}$ and phase $\beta$, as in the preceding discussion, resulting in the following master equation for the atomic density operator $\hat{\rho}$ :

$$
\frac{\partial \hat{\rho}}{\partial t}=\left(\frac{\partial \hat{\rho}}{\partial t}\right)_{\mathrm{FD}}+i Q\left[e^{-2 i\left(\omega_{o} t+\beta\right)}|3\rangle\left\langle 1\left|+e^{2 i\left(\omega_{o} t+\beta\right)}\right| 3\right\rangle\langle 1|, \hat{\rho}\right] .
$$

Here the first term $(\partial \hat{\rho} / \partial t)_{\mathrm{FD}}$ refers to Eq. (20) of Ref. [12(a)] written in the Schrödinger picture. In the second term, $Q$ is an effective two-photon Rabi frequency that involves the quadrupole moment times the square of the field $\left(Q \sim\left\langle e^{2} r^{2}\right\rangle E_{\mathrm{RO}}^{2}\right)$.

From Eq. (7), equations of motion for the atomic populations $\rho_{i i}(i=1,2,3)$ and coherences $\rho_{i j}(i \neq j)$ can be derived, from which solutions for steady-state excitation probabilities are found. In the limit that the angle $\vartheta$ over which the squeezed field is focused onto the atoms is small [strictly when $v(\vartheta) \ll 1$, with $v$ defined in Eq. (24) of Ref. [12(a)]] and in the absence of atomic saturation with all fields on resonance with their respective transitions, we find that

$$
\begin{aligned}
\rho_{33} \simeq & \left\{\frac{4 Q^{2}}{\alpha^{2}}\left[1+\frac{\sqrt{\alpha}|M|}{Q} \sin \xi\right]+\left[N^{2}+\frac{|M|}{\alpha}\right]\right\} v^{2}(\vartheta) \\
& +O\left(v^{3}\right)
\end{aligned}
$$

where $\alpha \equiv \gamma_{3} / \gamma_{2}$ (see Fig. 1). In the limit considered here, the phase $\xi$ is the same up to a constant phase shift as the argument $2 \beta+\phi$ of the phase-sensitive term in Eqs. (3) and (4), although this is not the case in general.

Note that for $Q=0$, Eq. (8) reduces to Eq. (23) of Ficek and Drummond [12(a)]. Indeed, in a previous experiment with $Q=0$ [14], we have shown that the nonclassical nature of the squeezed vacuum as manifest in the second term of Eq. (8) leads to a departure from the usual quadratic form with a linear dependence in the two-photon excitation rate predicted for $N \rightarrow 0$. By contrast, in this paper the focus of our attention is the first term of Eq. (8), which for large $Q$ (strong reference oscillator limit) becomes dominant, so that

$$
\rho_{33} \rightarrow \frac{4 Q^{2}}{\alpha^{2}}\left[1+\frac{\sqrt{\alpha}|M|}{Q} \sin (2 \beta+\phi)\right] v^{2}(\vartheta),
$$

which is of the same form as Eq. (4).

Turning now to our experimental scheme as depicted in Fig. 2, we associate the atomic states $(1 ; 2 ; 3)$ with the $\left(6 S_{1 / 2}, F=4 ; 6 P_{3 / 2}, F^{\prime}=5 ; 6 D_{5 / 2}, F^{\prime \prime}=6\right)$ levels of atomic cesium with transition frequencies $\left(\omega_{12}, \omega_{23}, \omega_{13}\right)$ and wavelengths $\lambda_{12} \simeq 852 \mathrm{~nm}, \lambda_{23} \simeq 917 \mathrm{~nm}$, and $\lambda_{13} \simeq 884 / 2 \mathrm{~nm}$, respectively. As described in Ref. [13], the cesium atoms are cooled and trapped in a magnetoptical trap (MOT), with the trapping lasers chopped at a rate of $4 \mathrm{kHz}$ to provide On and Off periods with and without the trapping fields. Our data acquisition of fluorescence from the $3 \rightarrow 2$ decay at $917 \mathrm{~nm}$ proceeds during the Off period by photon counting of the fluorescent intensity $F_{32}$, which is proportional to the excited-state population $\rho_{33}$. By following the set of procedures described in Ref. [14], we are assured that the sponta-

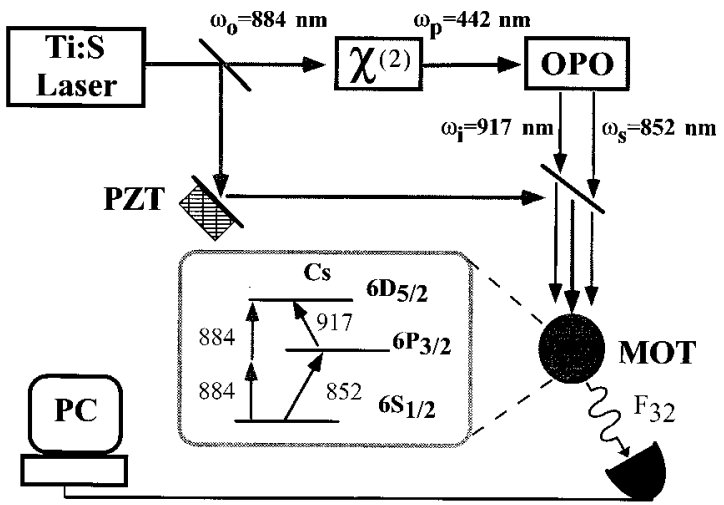

FIG. 2. Experimental setup.

neous parametric output from the nondegenerate optical parametric oscillator (ND-OPO) at the conjugate frequencies $\omega_{s}$ and $\omega_{i}$ and the Ti:sapphire laser frequency $\omega_{o}$ are peaked near the atomic transition frequencies $\omega_{12}, \omega_{23}$, and $\omega_{13} / 2$, respectively. More precisely, we estimate that the spectroscopic conditions $\left(\omega_{s} \simeq \omega_{12}, \omega_{i} \simeq \omega_{23}, \omega_{o} \simeq \omega_{13} / 2\right)$ are met to within $\pm(0.5,1.0,0.5) \mathrm{MHz}$, respectively.

Although the output of the ND-OPO should be squeezed, direct homodyne detection is not possible because the offset frequency $\Omega_{o} / 2 \pi=12 \mathrm{THz}$ requires photodetectors of the same bandwidth [Eq. (6)]. However, by virtue of Eq. (8), we can nonetheless investigate the asymmetric distribution of quantum fluctuations for a squeezed state [i.e., $M\left(\Omega_{o}\right) \neq 0$ ] by exploiting the atom as an ultrafast nonlinear mixer [6]. Towards this end, atoms in the MOT are illuminated with the output of the ND-OPO together with a copropagating reference oscillator beam at $\lambda_{o}=884 \mathrm{~nm}$. From measurements of the parametric gain for an injected signal beam at $852 \mathrm{~nm}$, we calculate that the powers of the squeezed fields at 852 and $917 \mathrm{~nm}$ are of order $1 \mathrm{pW}$, while the power of the coherent $\mathrm{RO}$ beam is adjusted to be around $5 \mathrm{~mW}$. The copropagating beams are focused with a waist of $10 \mu \mathrm{m}$ into the atomic Cs sample in the MOT [14], with the intensities of theses fields being $I_{852} / I_{s_{1}} \sim I_{917} / I_{s_{2}} \sim 10^{-3}$ and $I_{883} / I_{s_{0}} \sim 1$, where $I_{s_{i}} i=1,2,3$ are the saturation intensities for the $1 \rightarrow 2,2 \rightarrow 3$, and $1 \rightarrow 3$ transitions, respectively. To probe the excited-state population $\rho_{33}$, fluorescence $F_{32}$ from decay of the $6 D_{5 / 2}, F^{\prime \prime}=6 \rightarrow 6 P_{3 / 2}, F^{\prime}=5$, transition at $917 \mathrm{~nm}$ is collected with an $f \simeq 1$ imaging system and focused onto an avalanche photodetector, the output of which is counted and stored under computer control.

With the above intensities and for our system efficiencies, the counting rates are about $C_{\mathrm{sq}} \sim 1-2 \mathrm{~s}^{-1}$ due to the squeezed fields alone [corresponding to the second term in Eq. (8)] and about $C_{\mathrm{RO}} \sim 10^{4} / \mathrm{s}$ due to the $884 \mathrm{RO}$ field alone [the first term in Eq. (8)]. The phase $\xi=2 \beta+\phi$ is varied by sweeping $\beta$ as $\beta(t)=\beta_{o}+\omega_{m} t$ with a PZT in the path of the RO (Fig. 2). The expected visibility for the resulting modulation of $F_{32}$ based on the above counting rates is then $V \equiv C^{\mathrm{max}}-C^{\min } / C^{\mathrm{max}}+C^{\min } \simeq \sqrt{C_{\mathrm{sq}} / C_{\mathrm{RO}}} \simeq 10^{-2}$ for $C_{\mathrm{RO}}$ $\gg C_{\mathrm{sq}}$. To observe directly this modulation in $\rho_{33}$ versus $\beta$ requires that $\sqrt{C_{\mathrm{sq}}} \gg 1$, which for our counting rates demands observation intervals $\tau \sim 10-100 \mathrm{~s}$. Since the relative stability of phases of the reference oscillator and squeezed fields is not adequate for measurements of this duration, we 

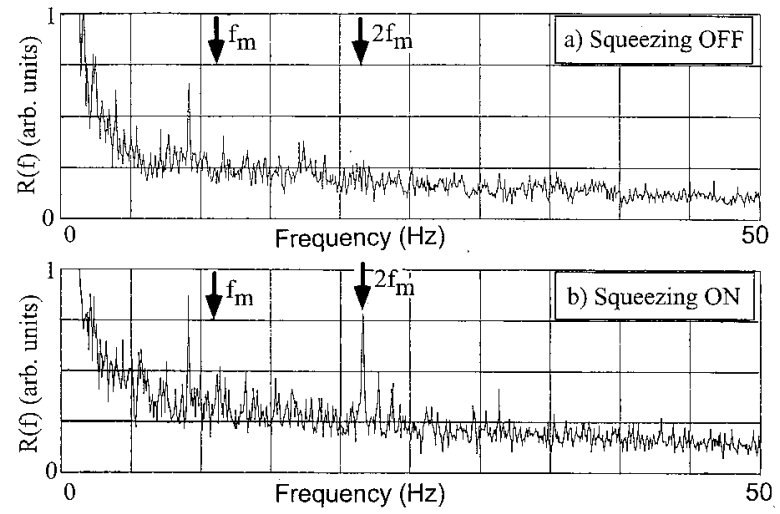

FIG. 3. Power spectrum $R(f)$ for the photocurrent due to the fluorescence $R_{32}$, which serves as a measure of the excited-state population $\rho_{33}$. (a) Without the squeezed fields at $\omega_{s, i}$ no modulation at either $f_{m}$ or $2 f_{m}$ is observed. (b) With the squeezed field from the ND-OPO at $\omega_{s, i}$, a peak at frequency $2 f_{m}$ appears in accord with Eq. (9) and reflects the field correlation function $M\left(\Omega_{o}\right)$.

follow an alternative strategy of modulating $\beta$ at a somewhat higher frequency $\omega_{m} \gg \tau^{-1}$ (typically $\omega_{m} / 2 \pi \simeq 10 \mathrm{~Hz}$ ) and then analyzing the fluorescence from the $3 \rightarrow 2$ decay not in time but in the Fourier domain.

An example of phase-sensitive detection of quantum fluctuations by this technique is given in Fig. 3, where we display the power spectrum $R(f)$ obtained from the Fourier transform of the photocurrent arising from the fluorescence $F_{32}(t)$. Shown in Fig. 3(a) is a reference spectrum with excitation of the MOT by the 884-nm field alone in the absence of the signal and idler fields from the ND-OPO, but with the phase $\beta(t)$ nonetheless modulated as described about, with $\omega_{m} / 2 \pi \equiv f_{m}=11 \mathrm{~Hz}$. No modulation at either $f_{m}$ or $2 f_{m}$ is visible above the noise floor, which is set by the Poisson fluctuations of the counting rate $C_{\mathrm{RO}}$ ("shot noise"). In contrast, in Fig. 3(b) the RO field at $884 \mathrm{~nm}$ together with the signal and idler fields at 852 and $917 \mathrm{~nm}$ simultaneously illuminate the Cs sample. Now there is a clear Fourier com- ponent in $R(f)$ at frequency $2 f_{m}$ corresponding to the modulation of $\rho_{33}$ as in Eq. (9) due to the field correlation function $|M|=\left|\left\langle\hat{E}_{917} \hat{E}_{852}\right\rangle\right|$. Hence, Fig. 3 represents the detection of phase-sensitive correlations for fields separated by $\Delta \omega / 2 \pi=\left|\omega_{852}-\omega_{917}\right| / 2 \pi \simeq 25 \mathrm{THz}$.

Unfortunately in and of itself, Fig. 3 does not represent unambiguous detection of a nonclassical field since our method does not provide a simple analog of the shot-noise reference level of homodyne detection. One possible path to circumvent this difficulty was discussed earlier. Alternatively, the magnitude of the visibility $V$ could be recorded as a function of incident flux, and in this way $M$ as a function of $N$ determined. Since for quantum (squeezed) fields $N<M \leqslant \sqrt{N(N+1)}$ while for classical fields $M \leqslant N$, distinguishing between functional dependences for $V$ vs $N$ would provide a convincing case for the nonclassical nature of the correlation function $M$. Indeed, very much in this spirit, the measurements reported here (as in Fig. 3) together with our earlier work (Ref. [14]) clearly demonstrate both the asymmetry of the phase-space distribution and the nonclassical character of the field emerging from the ND-OPO, although this is admittedly a rather arduous path.

In conclusion, we have demonstrated a technique for probing correlations between fields that are separated by large frequency intervals $(\Delta \omega=25 \mathrm{THz}$ ) not previously accessible via usual homodyne techniques. Our method is based on a scheme of quantum interference where the atoms act as ultrafast nonlinear mixers that translate to lowfrequency field correlations that exist with large separation $\Delta \omega$. Beyond the domain of quantum optics, our basic idea of utilizing atoms as nonlinear mixers for demodulation over large frequency intervals (tens of hundreds of $\mathrm{THz}$ ) can be generalized and extended into other areas, including frequency metrology for fundamental measurements and optical communications [6].

This work was supported by the Division of Chemical Sciences, Office of Basic Energy Sciences, Office of Energy Research, U.S. Department of Energy.
[1] J. Opt. Soc. Am. B 4(10) (1987), special issue on squeezed states of the electromagnetic field, edited by H. J. Kimble and D. F. Walls; Appl. Phys. B 55(3) (1992), special issue on quantum noise reduction in optical systems-experiments, edited by E. Giacobino and C. Fabre.

[2] H. P. Yuen and J. H. Shapiro, IEEE Trans. Inf. Theory 26, 78 (1980); H. P. Yuen and V. W. S. Chan, Opt. Lett. 8, 177 (1983).

[3] M. G. Raymer, J. Cooper, H. J. Carmichael, M. Beck, and D. T. Smithey, J. Opt. Soc. Am. B 12, 1801 (1995), and references therein.

[4] B. Yurke, Phys. Rev. A 32, 311 (1985).

[5] C. M. Caves and B. L. Schumaker, in Quantum Optics IV, edited by J. D. Harvey and D. F. Walls (Springer-Verlag, Berlin, 1986), pp. 20-30.

[6] N. Ph. Georgiades, E. S. Polzik, and H. J. Kimble, Opt. Lett. 21, 1688 (1996).
[7] B. R. Mollow, Phys. Rev. 175, 1555 (1968).

[8] H. P. Yuen, Phys. Rev. A 13, 2226 (1976); M. J. Collett and R. Loudon, J. Opt. Soc. Am. B 4, 1525 (1987).

[9] H. J. Kimble, in Fundamental Systems in Quantum Optics, edited by J. Dalibard, J. M. Raimond, and J. Zinn-Justin (Elsevier Science, Amsterdam, 1992), pp. 595-606.

[10] For the purpose of the present discussion, we assume that $N(\Omega)=N(-\Omega)$, which is valid if the ND-OPO has the same absorptive and dispersive properties at $\omega_{s, i}$.

[11] N. Ph. Georgiades, E. S. Polzik, and H. J. Kimble (unpublished).

[12] (a)Z. Ficek and P. D. Drummond, Phys. Rev. A 43, 6247 (1992); (b)43, 6258 (1992).

[13] N. Ph. Georgiades, E. S. Polzik, and H. J. Kimble, Opt. Lett. 19, 1474 (1994).

[14] N. Ph. Georgiades, E. S. Polzik, K. Edamatsu, H. J. Kimble, and A. S. Parkins, Phys. Rev. Lett. 75, 3426 (1995). 